\title{
Effets de l'addition d'iso-acides à la ration sur la fermentation ruminale, la dégradation in sacco de la matière sèche et la concentration de quelques paramètres sanguins chez la vache
}

\author{
JI Andries¹, BG Cottyn ${ }^{1}$, S De Keersmaecker², FX Buysse ${ }^{1}$ \\ 1 Station de Recherches sur l'Alimentation du Bétail, CLO-Gand, Scheldeweg 68, \\ 9231 Melle-Gontrode; 2 Faculté des Sciences Vétérinaires, Université de Gand, \\ Casinoplein 24, 9000 Gand, Belgique
}

\begin{abstract}
Summary - Three rumen-fistulated cows fed maize silage and concentrates were supplemented with either soybean oil meal or urea, associated or not with isoacids. The isoacids increased the volatile fatty acid concentration, lowered the ruminal ammonia concentration and in sacco dry matter disappearance and did not influence blood parameters.
\end{abstract}

Introduction - Les iso-acides auraient un rôle bénéfique sur la fermentation ruminale. De plus, une influence sur le métabolisme intermédiaire est quelquefois suggérée (Andries et al, 1987). Nous avons testé les effets d'un supplément d'isoacides sur la fermentation ruminale, sur la dégradation in sacco de la matière sèche (MS) et sur quelques paramètres sanguins.

Matériel et Méthodes - L'essai a été effectué sur 3 vaches en lactation, fistulées du rumen. Pendant 4 périodes consécutives de 21 j on a compare 4 traitements qui consistaient en une augmentation du niveau de la matière azotée totale d'une ration de base de 12 à $15 \%$ (par rapport à la MS) et ceci par addition respectivement de : $A$ : tourteau de soja; $B$ : tourteau de soja + iso-acides"; C : urée; D : urée + iso-acides*. La ration de base était composée d'ensilage de maïs $(33,4 \%$ MS) et d'un aliment concentré. Les vaches ont été nourries à volontó. Le rapport ensilage de maïs:concentré a été fixé à 50:50 pour 2 vaches, et à 70:30 pour la troisième. L'ensilage de maïs et les concentrés ont été distribués ensemble 2 fois par jour. L'urée a été mélangée à l'ensilage de maïs, les iso-acides ont été mélangés avec les concentrés. La composition du jus de rumen a été déterminée dans des échantillons prélevés à jeun et 3 et $8 \mathrm{~h}$ après le repas du matin, les 2 dernières journées de chaque période expérimentale. La détermination de la dégradation in sacco de la MS d'un foin, de l'ensilage de maïs et de la pulpe sèche a été effectuée les 2 dernières journées de chaque période. Par aliment et par vache, 4 sachets ont été incubés pendant 24 et $48 \mathrm{~h}$. La dernière journée de chaque période, du sang a été prélevé juste avant le repas du matin et 1, 2 et $6 \mathrm{~h}$ après, pour la détermination des teneurs en glucose et en corps cétoniques.

Résultats et Discussion - Le pH du jus de rumen et les proportions relatives des acides gras volatils (AGV) majeurs du rumen ont été semblables pour les 4 trai-

\footnotetext{
* $86 \mathrm{~g}$ d'une préparation commerciale, contenant environ $82 \%$ de sels de calcium des acides isobutyrique, 2 methylbutyrique, valérique et isovalérique
} 
Tableau I. Résultats expérimentaux '

\begin{tabular}{|c|c|c|c|c|}
\hline & \multicolumn{4}{|c|}{ Traitements } \\
\hline & $A$ & $B$ & $c$ & $D$ \\
\hline $\begin{array}{l}\text { Caractéristiques ruminales (moye } \\
\text { pH } \\
\text { conc des AGV (mmol/100 ml) } \\
\text { ac acétique (mol \%) } \\
\text { ac propionique (mol \%) } \\
\text { ac butyrique (mol \%) } \\
\text { iso-acides totaux (mol \%) } \\
\mathrm{NH}_{3}(\mathrm{mg} / 100 \mathrm{ml})\end{array}$ & $\begin{array}{l}\text { lèvemen } \\
6,4^{\mathrm{a} 2} \\
10,2^{\mathrm{ab}} \\
57,8^{\mathrm{a}} \\
19,6^{\mathrm{a}} \\
16,2^{\mathrm{a}} \\
4,6^{\mathrm{a}} \\
19,3^{\mathrm{a}}\end{array}$ & $\begin{array}{r}3 \text { et } 8 \mathrm{ha} \\
6,2^{\mathrm{a}} \\
11,3^{\mathrm{a}} \\
58,2^{\mathrm{a}} \\
19,5^{\mathrm{a}} \\
16,9^{\mathrm{a}} \\
4,8^{\mathrm{a}} \\
17,5^{\mathrm{a}}\end{array}$ & $\begin{array}{l}\text { le repas) } \\
6,3^{\mathrm{a}} \\
9,8^{\mathrm{b}} \\
59,1^{\mathrm{a}} \\
19,7^{\mathrm{a}} \\
16,8^{\mathrm{a}} \\
3,4^{\mathrm{b}} \\
22,0^{\mathrm{a}}\end{array}$ & $\begin{array}{c}6,3^{a} \\
10,4^{\text {ab }} \\
58,1^{a} \\
21,1^{a} \\
15,7^{a} \\
4,3^{a b} \\
16,6^{a}\end{array}$ \\
\hline $\begin{array}{l}\text { Dégradation in sacco de la MS (\% } \\
\text { foin } \\
\text { ensilage de maïs } \\
\text { pulpe sèche }\end{array}$ & $\begin{array}{l}59,3^{a b} \\
67,1^{a} \\
91,8^{a}\end{array}$ & $\begin{array}{l}45,5^{\mathrm{a}} \\
55,8^{\mathrm{a}} \\
84,6^{\mathrm{a}}\end{array}$ & $\begin{array}{l}54,7^{\mathrm{b}} \\
61,0^{\mathrm{a}} \\
90,0^{\mathrm{a}}\end{array}$ & $\begin{array}{l}50,7^{\mathrm{ab}} \\
64,5^{\mathrm{a}} \\
87,7^{\mathrm{a}}\end{array}$ \\
\hline $\begin{array}{l}\text { Paramètres sanguins } \\
\text { glucose (mmol/ }) \\
\text { ac acéto-acétique (mmol/l) } \\
\text { ac } \beta \text {-hydroxybutyrique (mmol/l) }\end{array}$ & $\begin{array}{l}3,2^{a} \\
0,34^{a} \\
2,3^{a}\end{array}$ & $\begin{array}{l}3,0^{\mathrm{a}} \\
0,09^{\mathrm{a}} \\
1,1^{\mathrm{a}}\end{array}$ & $\begin{array}{l}3,4^{\mathrm{a}} \\
0,07^{\mathrm{a}} \\
1,0^{\mathrm{a}}\end{array}$ & $\begin{array}{l}3,3^{\mathrm{a}} \\
0,04^{\mathrm{a}} \\
0,8^{\mathrm{a}}\end{array}$ \\
\hline
\end{tabular}
1 Tous les résultats sont statistiquement traités par une analyse de variance. ${ }^{2}$ Les moyennes d'une même ligne in-
dexées par la méme lettre ne sont pas significativement différentes $(P>0,05)$.

tements (tableau I). Le traitement $\mathrm{C}$ a provoqué un abaissement significatif de la concentration des iso-acides par rapport aux traitements $\mathrm{A}$ et $\mathrm{B}$, probablement à cause de la plus faible teneur en acides aminés précurseurs de la ration maïsurée. L'addition d'iso-acides dans le traitement $D$ a rétabli leur proportion à un niveau proche de celui des traitements $A$ et B. L'addition d'iso-acides a accru la concentration des AGV et abaissé celle de $\mathrm{NH}_{3}$, ce qui suggère une augmentation de l'activité fermentaire associée à une protéosynthèse accrue, phénomène qui a déjà été démontré antérieurement (Russell et Sniffen, 1984). En revanche, les résultats de dégradation in sacco indiqueraient que les iso-acides ont freiné la dégrada- tion ruminale des substrats testés, alors que leur rôle bénéfique sur la digestion des parois cellulaires a souvent été mentionné. Aucun paramètre sanguin n'a été modifié significativement. Les iso-acides n'ont donc pas contribué au métabolisme glucogénique et cétonique.

En conclusion, les iso-acides ont stimulé la concentration des AGV, ont diminué la teneur en $\mathrm{NH}_{3}$ et influencé négativement la dégradation in sacco de la MS. En plus ils n'ont pas eu d'effet sur la teneur en glucose et en corps cétoniques du sang.

Andries Jl, Buysse FX, De Brabander DL, Cottyn BG (1987) Anim Feed Sci Technol 18, 169-180

Russell JB, Sniffen CJ (1984) J Dairy Sci 67, 987-994 